\title{
Author index to volume 37
}

Key to abbreviations:

(BR) Book Review; (CB) Cententary of the Birth; (C) Clinical case of the Month; (CR) Case Report; (E) Editorial; (H) Hypothesis; (L) Letter; (O) Obituary; (P) Profile; (R) Review; (RL) Reply to letter; (SR) Scientific Review; (SC) Short Communication

Abel R 14

Able AC 50, 666 (RL)

Adkins RH 485, 765

Aebi M 392 (C)

Ağildere M 305 (CR)

Ahmed YS 25

Aiello I 258

Aito S 617 (C)

Akaboshi K 58

Akdemir H 29

Akman MN 305 (CR), 332,638

Al-Khodairy AT 142 (CR)

Aleksandrowicz R 710

Alencar HMP 793

Almenoff PL 279

Anand J 737 (CR)

Annoni JM 142 (CR)

Aréchaga C 450 (CR)

Asazuma T 753

Asensi JM 450 (CR)

Asensi V 450 (CR)

Aufdemkampe G 123

Bacher T 172

Bach-y-Rita P 465 (SR)

Baer GA 239 (C)

Balvis B 54

Baltas C 453 (L)

Barbalata L 284

Barbeau H 245, 430

Barber DB 50, 666

(RL)

Barin K 40

Barros TEP 89 (C), 166 (C)

Batavia AI 140 (CR)

Batavia M 140 (CR)

Bauman WA 485, 601

(SR), 765

Baumberger ME 239 (C)

Belgrano E 258

Bell G 264

Bell MH 653

Beneforti P 440

Beneton C 370 (CR)

Bengi R 638

Bentolila S 548
Bergstrom EMK 838
Bernard PL 129
Bernardi M 336
Betz RR 585
Bhutani R 560
Biering-Sørensen F 89
(C), 166 (C), 299 (SC),
680 (C)
Bierzyńska-Macyszyn G
710
Blanksby BA 553
Blitvich JD 553
Bohlman HH 166 (C)
Boisson D 370 (CR)
Borwell B 211
Bötel U 239 (C), 680 (C)
Boudaoud L 548
Boutsen Y 301 (CR)
Bowers DM 508
Bowsher D 235 (SR)
Bracken MB 833
Brink D 421
Brooks CA 284
Brown K 653
Buczynski AZ 749 (C)
Bunge RP 6
Bükte Y 448 (SC)
Burnham R 264
Burnside IG 25
Bussel B 548
Butt L 325 (C)
Bygrave MA 6

Campbell IG 772

Capen DA 166 (C)

Cardenas DD 191

Cardone C 416

Carlier R 548

Carlstedt T 811

Carroll M 325 (C)

Carron JA 760

Carton JA 450 (CR)

Casadevall N 548

Castellano V 336

Castro C 416

Cawley MF 705

Ceviz A 448 (SC)
Chapman JR 444

Charlifue SW 183

Chilibeck PD 264

Chino N 58

Clowers DE 204

Conroy L 624

Coratella D 336

Coskun E 480

Cotler J 166 (C)

Craig A 345

Craig JJ 275

Craven M 508

Crook H 569

Crosbie J 780

Cullheim S 811

Curt A 157

Curtis KA 421

Cuxart A 351

d'Aloia G 258

D'Andrea M 617 (C)

da Graça JRV 793

Dallmeijer AJ 96

Dalyan M 191

Delaere KPJ 208, 373 (CR)

Deltombe T 301 (CR)

Denis F 392 (C)

Derry FA 110

Desai KI 866 (CR)

Deutsch A 717

Di Lorenzo N 617 (C)

Dickson H 345

Didyk T 421

Dietz V 157, 595 (L)

Dimitrijevic MR 531 (SR)

Dinsmore WW 110

Diokno AC 1 (C)

Dion S-A 730

Ditunno Jr JF 336, 402

Dizien O 548

Dollfus P 218, 231 (BR)

Dons K 166 (C)

dos Santos AA 793

Douglas GA 553

Dreikorn K 196

Dudley GA 251
Edmond P 501

Egnatchick JE 862 (CR)

Ejeskar A 475 (C), 871

(RL),

El Masry WS 1 (C), 153 (O), 617 (C), 538 (C)

Ersoz G 644

Exner G 239 (C)

Farooque M 696

Fellows GJ 749 (C)

Ferguson KA 800 (CR)

Fernández M 54

Ficicilar H 644

Figoni SF 685

Finkelstein JA 444

Finnis D 211

Finsterer J 592 (SC)

Folgering HThM 575

Forner-Cordero I 522 (CR)

Frankel H 461 (CB), 526 (BR), 838, 847

Franzoi AC 416

Fraser WD 760

Freebourn TM 50, 666 (RL)

Freehafer AA 871 (L)

Freund M 62 (CR), 617 (C)

Fromm B 239 (C), 617 (C)

Frondizi D 68 (CR)

Fujimura Y 580, 753

Fujiwara T 58

Gaebler C 33, 377 (RL)

Gallagher JA 760

Gambino R 485

Garcia J 65 (CR)

García-Manzanares MD $522(\mathrm{CR})$

Gardner BP 680 (C)

Gazzani F 336

Gean K 421

Gepstein R 215

Geraghty TJ 508 
Gerard B 191

Gerhart KA 183, 284

Gerner HJ 14, 62 (CR), 151 (O), 239 (C), 617 (C)

Gervais M 730

Giannantoni A 308 (RL)

Gilliard C 301 (CR)

Gisbert-Vicens J 522 (CR)

Glasauer FE 862 (CR)

Glass CA 110, 183, 325 (C)

Goel A 866 (CR)

Goin JE 289

Gondim FAA 793

Gothe B 71 (L)

Granat MH 501

Grangeon C 858

Granger CV 717

Gray WJ 275

Graziani V 402

Grimm DR 279, 601 (SR)

Grundy DJ 211

Guiducci A 68 (CR)

Gupta SK 726

Guptar PM 658

Gustin T 301 (CR)

Hagen DL 508

Hall J 421

Hamelin M 245

Hamilton LR 136

Hancock K 345

Hanson P 301 (CR)

Hara Y 58

Harley J 455 (BR)

Harris M 392 (C)

Harris P 463 (CB)

Harvey AS 786

Harvey LA 780

Hashizume Y 224 (CR)

Henderson NJ 838

Hentz VR 475 (C)

Herbert R 485

Hernández D 65 (CR)

Hillegass EA 251

Hirabayshi K 753

Hollander AP 96

Holst-Nielsen F 475 (C)

Holtz A 696

Hooper M 421

Hopman MTE 575

Hoque MF 858

Houtman S 575

Huch K 648

Hundt G 239 (C)
Iannotti F 231 (BR)

Iborra J 351

Ikegami Y 358

Illis LS 310 (BR), 378

(BR), 526 (BR), 596

(BR), 665 (BR), 806

(BR)

Islekel H 480

Iwatsubo E 1 (C), 749

(C)

Jaffray DC 377 (L)

Jain AK 362

Jaovisidha S 453 (RL)

Jastrzab G 221 (CR)

Jayakumar PN 47

Jeon J 264

Jessiman TL 228 (CR)

Jones PRM 838

Juan García FJ 54

Kahn NN 601 (SR)

Kakulas BA 79 (SR), 595 (RL)

Kalaivant PL 47

Kalita J 297, 658

Kamper D 40

Kaplan PE 805 (L)

Karakücük EI 29

Karatas M 305 (CR), 638

Karlsson A-K 383 (SR), 494, 805 (RL)

Kawauchi Y 269

Kawazu T 542

Kearney J 705

Keith MW 475 (C)

Kelly SR 211

Kemp BJ 485

Kennedy P 136, 183, 847

Khor KE 221 (CR)

Khosla VK 726

Kilinç S 305 (CR), 332, 638

Klekamp J 103

Klos J 421

Kluger P 648

Ko H-Y 402

Kobetic R 800 (CR)

Koc RK 29

Kounis NG 453 (L)

Kovindha A 218

Koyanagi T 1 (C)

Krassioukov AV 6

Kreuter M 325 (C)

Krishnan KR 594 (L)

Kumar A 726

Kumar S 362

Kunz U 648
Kutscha-Lissberg F 33

Ladziński P 710

Lajoie Y 245

Laramée M-T 730

Lavara-Perona MC 522 (CR)

Lawton NF 73 (BR)

Lazzeri M 440

Leitner J 215

Lentell G 421

Lemaire ED 430

Lesina S 421

Lesser M 279

Levendoḡlu F 332

Li S 820

Liguori G 258

Lion S 54

Lissiani A 258

Little J 680 (C)

Little JW 402

Lobben B 515

Lortat-Jacob S 548

Macaluso A 336

Macellari V 336

Madersbacher HG 196, 749 (C)

Maier R 33

Maira G 68 (CR)

Maislin G 717

Majchrzak H 710

Makino K 542

Maliszewski M 710

Maloney P 485

Mamoli B 592 (SC)

Mansour P 760

Maradona JA 450 (CR)

Marchesi DG 166 (C)

Marchetti M 336

Marino RJ 289, 717

Marsolais EB 800 (CR)

Martin EME 353 (RL)

Martinez M 65 (CR)

Mastronardi L 68 (CR)

Mathuriya SN 726

Matsunaga S 20

Maximin A 71 (L)

Maynard C 653

Maytom MC 110

McCann JP 275

McCarthy S 310 (BR)

McCreavy DT 760

McDicken IW 760

McElroy GK 553

McEvoy RD 71 (RL)

McKenna K 624

McLean DE 705
Meinecke F-W 151 (O)

Meiners T 14

Mellick DC 284

Melis EH 430

Melwill R 680 (C)

Menkü A 29

Mertens P 370 (CR)

Meyer Jr PR 392 (C)

Michielsen DP 201

Middleton JW 508

Mirza S 444

Misra UK 297 (SC), 658

Mizuno J 224 (CR)

Mizushima T 542

Momose H 89 (C)

Monji K 542

Montoto A 54

Moraes AC 538 (C)

Moraleda S 65 (CR)

Morio Y 853

Mossberg K 569

Mrkonjic L 33

Mulcahey M-J 585

Murali T 47, 737 (CR)

Muzumdar DP 866 (CR)

Nagashima H 853

Nair KPS 47

Nakagawa H 224 (CR)

Nakamura M 580

Nakanisi K 20

Nardi M 68 (CR)

Naumann CP 239 (C)

Nawata K 853

Nesathurai S 228 (CR)

Nielsen K 299 (SC)

Nielsen OA 680 (C)

Niethard FU 172

Nisolle JF 301 (CR)

Noreau L 730

North NT 671 (SR)

O'Shaughnessy D 665 (BR)

Oberg S 325 (C)

Ogata H 542

Ohl DA 89 (C), 527 (RL)

Ohry A 325 (C)

Ohta H 680 (C)

Ohta K 580

Okawa H 542

Öktem IS 29

Olsson Y 696

Oña M 450 (CR)

Oo T 117, 147 (SC), 663

Orr M 110

Osterloh IH 110 
Øyhaugen S 515

Ozates M 448 (SC)

Özkan Ü 448 (SC)

Özker R 332, 638

Pacillas B 421

Padankatty BS 47

Paeslack V 172

Pagès E 351

Papadaki PJ 453 (L)

Paramijt 726

Park C 538 (C)

Parnianpour M 40

Parsch D 62 (CR)

Parsons KF 594 (L)

Pasin M 644

Patak S 569

Pathak A 726

Pearce JMS 741 (SR)

Pellicanò G 617 (C)

Pentland W 786

Perkash I 749 (C)

Peterson WP 284

Pinter MM 531 (SR)

Plancke HRF 208

Polando G 800 (CR)

Pons C 208

Poulain M 129

Poyares DL 634

Prasad VSSV 560

Price MJ 772

Pucket WR 6

Puhl W 648

Rajeev K 726

Ravenscroft A 25

Ravishankar D 47

Reed K 858

Reger S 40

Richards JS 325 (C)

Richardson W 653

Richter R 196

Risling M 811

Rocha FT 89 (C)

Rode G 370 (CR)

Rodrigues CL 793

Rodríguez A 54

Rogers B 847

Rola FH 793

Rossier AB 666 (L)

Rothwell AG 475

Roussi J 548

Royo A 65 (CR)
Rutkowski SB 325 (C), 508

Sajkov D 71 (RL)

Sakou T 20, 269

Salvador S 54

Samii M 103

Sánchez J 54

Sánchez-Ponce G 522 (CR)

Sari I 448 (SC)

Sartoris DJ 453 (RL)

Sau G 258

Schaafsma RJH 373 (CR)

Schanke A-K 515

Schechter C 485

Schiltenwolf M 172

Schmitt 370 (CR)

Schurch B 666 (L)

Schwartz A 560

Schwartz ML 560

Sedgwick EM 73 (BR)

Sett PK 117, 147 (SC), 375 (SC), 594 (L), 663 (SC), 760

Sgantzos M 453 (L)

Sharkey PW 560

Sharma BS 726

Shashidharan M 211

Shepard MJ 833

Shewmon DA 313 (H)

Shiba K 538 (C)

Shimada K 410

Short DJ 838

Shrosbree R 680 (C)

Silver JR 308 (L)

Simsek M 448 (SC)

Singas E 279

Singh G 147 (SC), 375

(SC), 594 (L), 663 (SC), 760

Siracusano S 258

Sivaraman Nair KP 737 (CR)

Smit C 14

Smith BT 585

Smith D 485

Smith MD 110

Smith T 786

Soni BM 117, 147 (SC), 375 (SC), 594 (L), 663 (SC), 760
Sønksen J 89 (C)

Sözay S 638

Speer S 847

Spinelli M 440

Spungen AM 485, 601 (SR), 765

Staal M 150 (BR)

Stener S 258

Stien R 527 (L)

Stineman MG 717

Stöhrer M 196, 749 (C)

Sullivan MF 378 (BR)

Süzer T 480

Suzuki N 753

Tahta K 480

Tajima F 542

Tallis RC 455 (BR)

Taly AB 47, 737 (CR)

Tator CH 538 (C), 820

Teshima R 853

Tewari MK 726

Theunissen PHMH 373 (CR)

Tokioka T 410

Topor MA 569

Torre M 336

Torres-Moreno R 430

Toyama Y 753

Triolo RJ 800 (CR)

Tromans AM 211

Tsuzuku S 358

Tufik S 634

Tuli SM 362

Túlio de Mello M 634

Turini D 440

Tyner TM 421

Uebelhart D 142 (CR)

Uijl SG 575

Vachon J 730

Vaidyanathan S 147

(SC), 375 (SC), 594

(L), 663 (SC), 760

Vallée C 548

van der Woude

LHV 96

van Dijk D 123 van Kamp GJ 96

van Langeveld S 123

Varray A 129

Vasudev MK 47

Vazquez MJ 65 (CR)

Vècsei V 33

Veerendra Kumar M 47

Velan GJ 215

Vest Hansen H 299 (SC)

Vinet A 129

Wagner GS 653

Walker J 786

Washburn RA 685

Watanabe M 580

Waters RL 485, 538 (C), 765

Watt JWH 117, 147 (SC), 663 (SC)

Weed H 40

Wehnert J 196

Weiss C 264

Weitzenkamp DA 183

Westergren H 696

Whiteneck GG 284

Willman C 569

Wing P 392 (C)

Wlodarski B 760

Wolańska-Karut J 710

Wyndaele JJ 1 (C), 201, 596 (BR)

Yabe K 358

Yang CC 204

Yato Y 580

Yavuzer S 644

Yone K 269

Yorgancioglu R 644

Yu WR 696

Zäch G 239 (C)

Zachary L 421

Zanollo A 440

Zavras GM 453 (L)

Zigler JE 166 (C) 